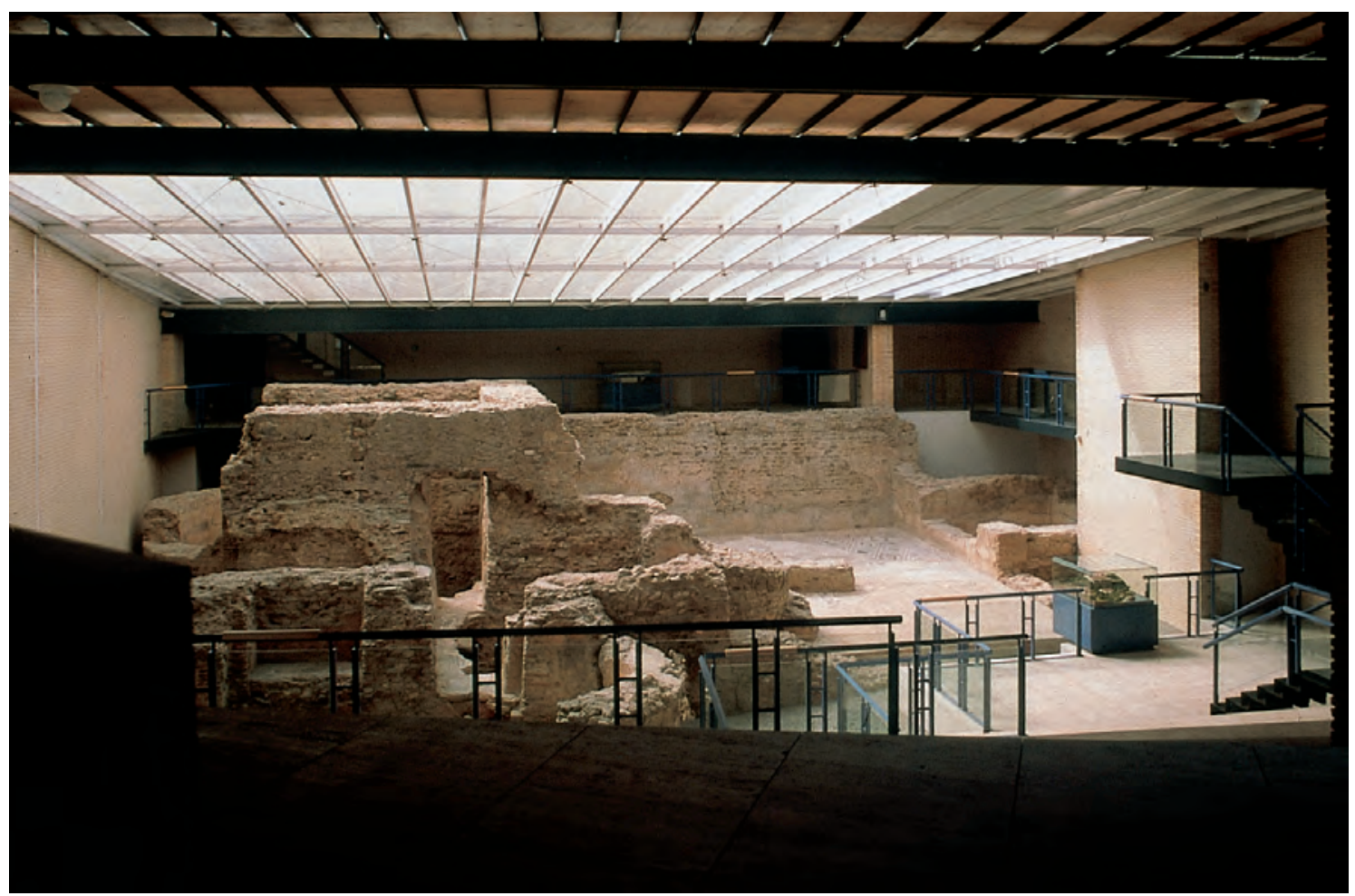

Primera visión que el espectador tiene de las termas al entrar por la puerta principal (foto: Antoni González. Septiembre 1998)

\title{
Consolidación y protección de las termas romanas de Sant Boi de Llobregat (Barcelona)
}

Antoni González *

A pesar de los avatares sufridos por sus fábricas, las termas romanas de Sant Boi se cuentan entre las mejor conservadas de Cataluña. Los criterios con los que el SPAL acomete su restauración, tras un riguroso estudio pormenorizado e interdisciplinar, son: la conservación y puesta en valor de sus restos; la protección de las mismas mediante un edificio que facilite la visita; y la disposición de gradas y un recorrido externo a los restos para evitar su deterioro y permitir un uso didáctico del conjunto.
Consolidation and protection of the Roman baths at Saint Boi de Llobregat. In spite of the hardships their fabrics have undergone, the thermal baths at Sant Boi are among the best conserved in Catalonia. The criteria followed by the SPAL in their restoration works, after a thorough interdisciplinary study, are the following: conservation of the remains; protection of the ensemble by means of a building to make visiting the site easier and the addition of steps and a route around the outside of the remains to prevent deterioration and permit the monument to be given a didactic use. 
En la antigüedad, el altozano ocupado ahora por el centro histórico del municipio de Sant Boi presidía la desembocadura de un río, el Llobregat, por lo que constituía un lugar estratégico para proteger un puerto, ventaja que favoreció su ocupación humana. El primer asentamiento conocido data de mediados del siglo IV aC y corresponde a un poblado de iberos layetanos. Doscientos años después, el lugar pertenecía al territorio adyacente a la ciudad de Barcino y en él se asentaba una villa romana formada por la residencia del propietario y su familia, las dependencias agrícolas y las tierras de cultivo. Gracias a la exportación de sus excedentes agrícolas (consta que desde el año 30 aC producía un vino que se vendía a las Galias e Italia), la villa se enriqueció y, hacia el año 200 dC, se amplió su área residencial, se construyó el edificio termal y se llevaron a cabo numerosas reformas en las demás dependencias.

\section{Las termas romanas}

En época romana, el baño era una costumbre generalizada que, en un principio, tenía lugar en instalaciones públicas llamadas termas. En ellas, además de cumplirse las funciones higiénicas y terapéuticas que les eran propias, se desarrollaba buena parte de la vida social y servían de salón de belleza. Más tarde, en las residencias privadas de categoría, como la de Sant Boi, se incorporaron estancias termales más o menos suntuosas, cuyo uso se extendió a lo largo del Alto Imperio.

Se accedía a las termas por el apodyterium o vestuario, en el que los bañistas cambiaban sus vestiduras por una pieza de tela sujeta a la cintura y su calzado por unas zapatillas de madera. De ahí se pasaba al tepidarium, donde se aclimataban al calor (entre $25^{\circ}$ y $30^{\circ}$ ) y untaban su piel con aceites perfumados. Después entraban en el laconicum (en el caso de las termas más antiguas) o el sudatorium, salas en las que el cuerpo transpiraba, en un ambiente seco o húmedo, a unos $80^{\circ}$. El laconicum se calentaba con braseros y el sudatorium mediante un hypocaustum, un sistema subterráneo de calefacción por aire caliente que, proveniente de un horno, pasaba bajo el suelo de las estancias (denominado suspensura, apoyado en pilarcillos de ladrillo, pilae, que descansaban sobre un pavimento inferior o area). El aire caliente podía también transmitirse a través de tubuli o tegulae mammatae (conductos de piezas de arcilla adosadas a las paredes), que calentaban los muros y permitían el tiro. En el caldarium, la siguiente sala, se registraba una humedad cercana al $80 \%$ y una temperatura de $55^{\circ}$; acostumbraba a contener una bañera de agua caliente $y$, a veces, una pila con agua fría situada en una exedra en la que se podían hacer abluciones. Antes de salir del recinto, los bañistas permanecían en la piscina del frigidarium para cerrar los poros de la piel. En las termas privadas, mientras el propietario y los invitados completaban el circuito, el servicio alimentaba con madera los hornos situados en una dependencia anexa.

En las termas públicas, que solían duplicar sus instalaciones para que pudieran ser utilizadas simultáneamente por hombres y mujeres, los usuarios, al final del recorrido, podían realizar ejercicios físicos en una palestra anexa.
1. Estado de las termas al inicio de los trabajos (foto: Jordi Isern, 30.05.1991)

2. Restitución ideal del edificio termal en la época del Bajo Imperio (dibujo: Txetxu Sanz. SPAL)

3. Sección transversal de las estancias cálidas de las termas (praefurnium, caldarium, sudatorium y tepidarium) (dibujo: Txetxu Sanz. SPAL)
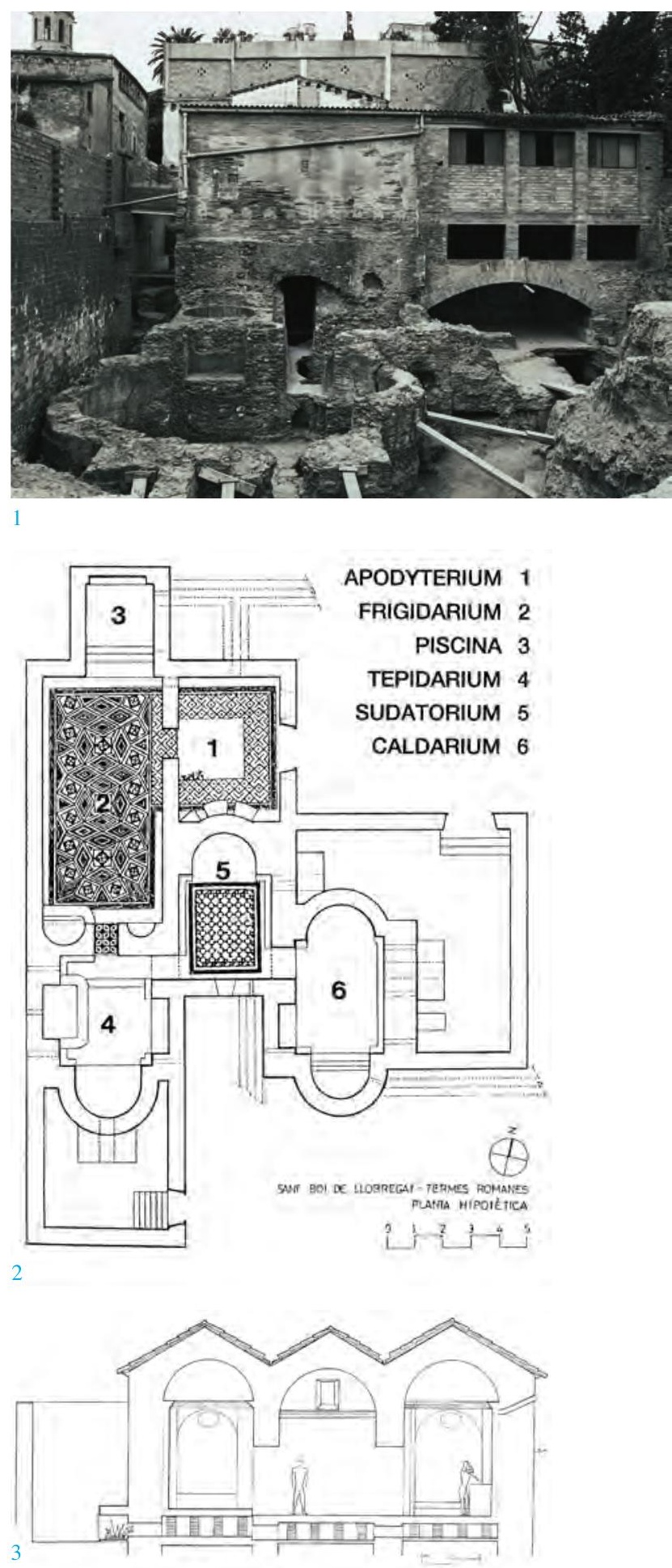


\section{La nueva intervención de la Diputación}

En 1988, el nuevo Ayuntamiento, consciente de la gravedad de aquel abandono y de la conveniencia de que las termas pudiesen ser visitadas, solicitó a la Diputación que volviese a intervenir con el asesoramiento técnico y científico y el apoyo económico necesarios para afrontar las complejas obras precisas. Aceptada la solicitud, el Servicio de Patrimonio Arquitectónico Local se encargó de las tareas de restauración, protección y exposición pública de los restos. ${ }^{4}$

La segunda etapa de intervención comenzó en 1989 con los estudios previos de carácter histórico, que incluyeron una investigación documental con el fin de precisar el alcance de las intervenciones anteriores y nuevas campañas de estudios arqueológicos. Éstos tuvieron como objeto determinar la extensión y situación de las ruinas en el solar (y la probable continuidad del yacimiento fuera de la finca municipal), así como delimitar el área exacta que había sido ya objeto de las investigaciones precedentes, para establecer una secuencia estratigráfica que interrelacionase la totalidad del yacimiento, y, si era posible, fechar el edificio, tarea entonces pendiente. Por último, se trató de hacer una restitución hipotética de la construcción primitiva, bien para proceder a su reconstrucción parcial, si así se decidía, o bien para explicar a los visitantes, mediante maquetas u otros procedimientos didácticos, cómo había sido.

Los trabajos de excavación incluyeron, además de las zonas anteriormente excavadas, la parte del yacimiento aún intacta (el solar situado a poniente de los restos, que aportó materiales que permitieron datar el recinto), y el entorno inmediato por levante y mediodía, donde fue posible una excavación más extensiva, aunque gravemente condicionada por la seguridad de los edificios o vías que rodeaban las termas. Finalmente, se hicieron una prospección por georádar, una serie de sondeos estratigráficos en zonas adyacentes a los restos y trabajos de exploración arquitectónica de éstos, y se procedió a desmontar las edificaciones construidas en los años sesenta para proteger el tepidarium y el frigidarium.

\section{Los criterios de la intervención}

Restaurar, proteger y exponer al público los restos romanos fueron, como he expuesto, los objetivos señalados en esta intervención del SPAL. Pero precisar cómo hacerlo no fue inmediato y se fue definiendo paralelamente a los trabajos de investigación; incluso se redefinió varias veces en el curso de la obra posterior (condicionada a la postre por una ampliación del solar inicial), en un proceso que no fue ni lineal ni sencillo.

Las respuestas a los tres objetivos estaban muy condicionadas unas con otras y ninguna tenía un punto de partida diáfano: las opciones de cómo restaurar los restos (consolidarlos únicamente, reconstruirlos según datos científicos, eliminar o no de ellos las aportaciones de las restauraciones anteriores, etc.) estaban condicionadas al cómo exponer esos restos, y viceversa (si el espectador debía acceder a ellos para pasearse y comprender así su espacio original; o si debía contemplarlos desde fuera directamente y tal como eran, o reconstruidos virtualmente mediante artilugios tecnológicos, etc.); y, en ambos casos, las decisiones estaban, a su vez, condicionadas por cómo proteger, tanto a los restos

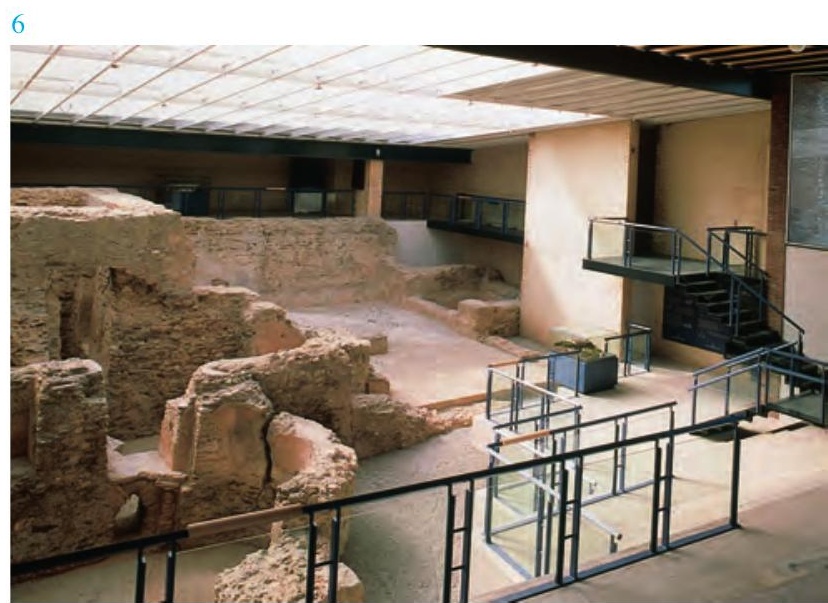

7
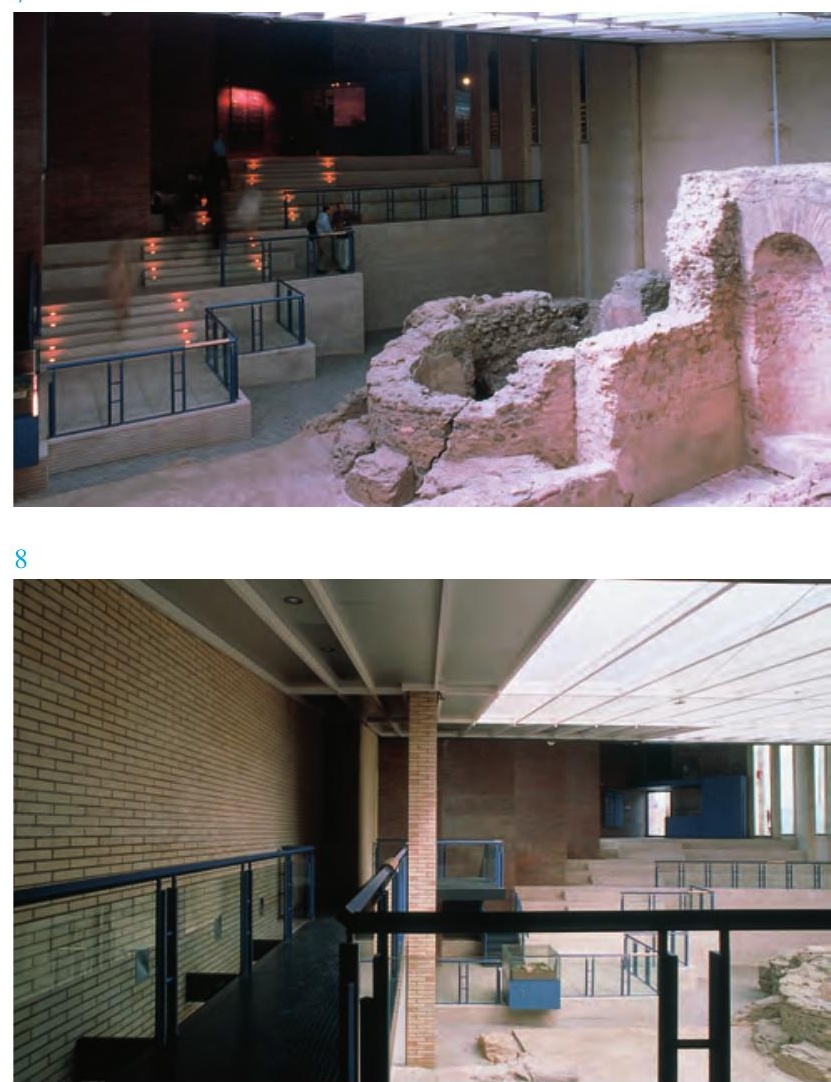
9. Vista de las estancias cálidas de las termas después de la restauración, iluminadas a través de la lucerna (foto: Montserrat Baldomà. SPAL. 10.09.1998)

10. Aspecto de las termas una vez restauradas. En primer término, la piscina y el mosaico del frigidarium (foto: Montserrat Baldomà. SPAL. 10.09.1998)

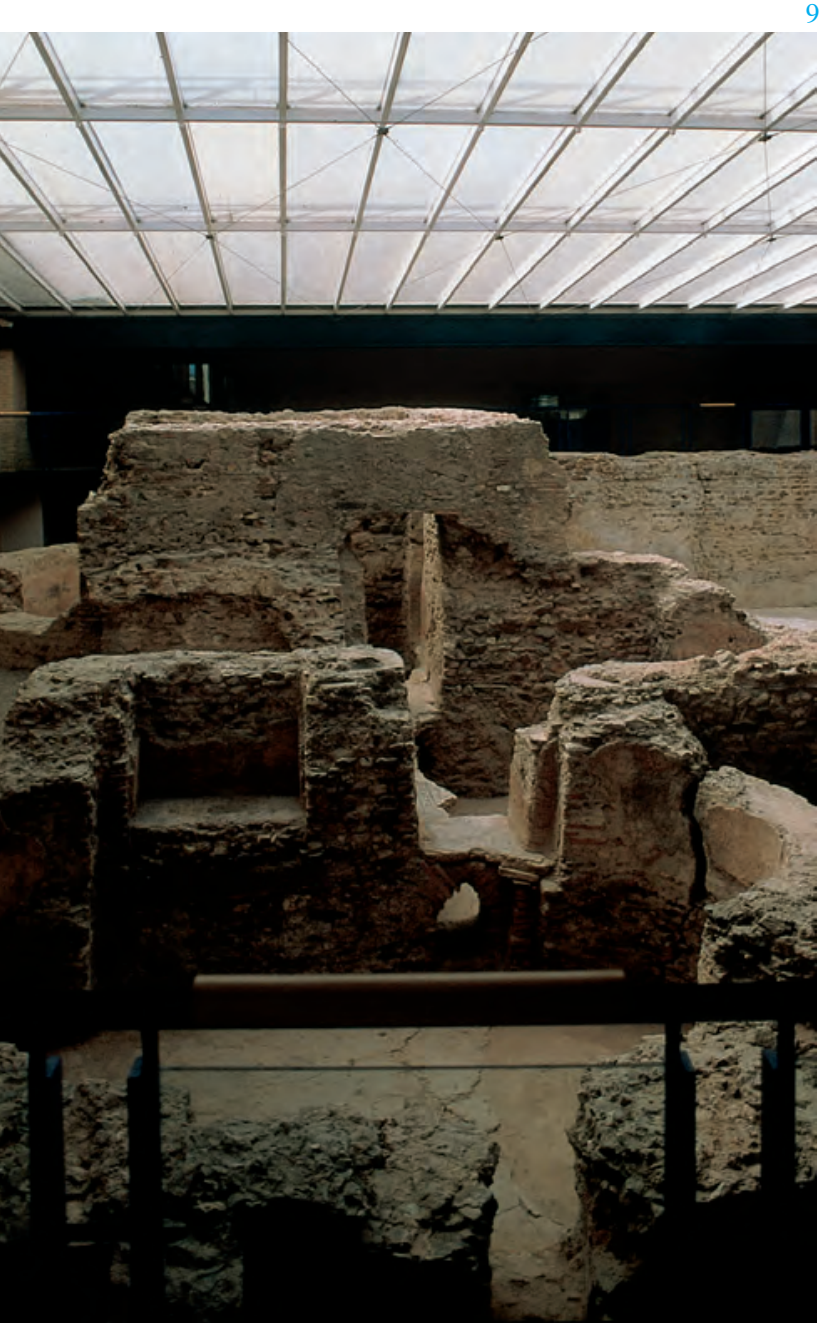

como a los visitantes (mediante un edificio climatizado, una simple cubierta, una sala anexa a un museo o un museo con un programa autónomo, etc.). A su vez, el carácter de esta instalación no dependía únicamente de planteamientos conceptuales, sino también de factores organizativos, técnicos y administrativos ajenos (ya que el presunto museo, o lo que finalmente fuera aquello, dependía del municipio, y éste cambió varias veces de responsables y de pareceres).

El conjunto final de los criterios establecidos, resultado de decisiones sucesivas (condicionadas además todas, como se ha dicho, por avatares de diverso tipo), fue éste: en primer lugar, conservar los restos de las termas (una vez limpiados, consolidados y puestos en valor) en la forma y el volumen en que habían llegado a nuestros días, sin eliminaciones ni añadidos (aunque, por supuesto, "crecidos", al haberse sacado a la luz restos no excavados con anterioridad); en cuanto a la visita, establecer un recorrido externo a los restos, para evitar su deterioro, y confiar la comprensión de su historia y su arquitectura a procedimientos didácticos tradicionales; y, por último, en cuanto a la protección, levantar un edificio de espacio único que permitiera la conservación de los restos y facilitara la visita; un edificio no concebido como museo sino como sala monográfica de una instalación de ámbito mayor (en cuanto a contenido e instalaciones), y, en consecuencia, con un programa exclusivo de visita, sin otro tipo de servicios o actividades, lo que permitía considerarla como una sala semiabierta, no climatizada.

Estos criterios programáticos encerraban otros, menos explícitos y más conceptuales: la arquitectura histórica a proteger y mostrar (el auténtico monumento, los restos de las termas) y la nueva arquitectura (el edificio protector) debían diferenciarse y respetarse mutuamente sin ambigüedad. Ni el monumento podía ser agredido en nada por la arquitectura nueva (ciertamente, subsidiaria de él), ni ésta podía estar condicionada estéticamente por aquél. Y el espectador debía intuir esa diferencia y respeto, sintiendo (sumadas, pero no confundidas) las emociones que pudieran producirle los restos históricos en cuanto tales (como reliquia, como documento) y, por otra parte, la nueva arquitectura (con sus posibles valores espaciales, formales y de uso).

\section{La restauración de las ruinas}

A pesar de los avatares sufridos por su fábrica, la de Sant Boi es una de las instalaciones termales romanas mejor conservadas de Cataluña (la altura de sus muros, por ejemplo, en ciertos puntos llega a los 4,80 m por encima del pavimento inferior del hipocausto, hecho nada habitual). Han llegado hasta nosotros, por otra parte, seis de las siete salas, en las que permanecen elementos muy significativos. El apodyterium conserva restos del pavimento de mosaico decorado con una orla con flores y una cenefa en forma de greca, con decoración geométrica realizada en blanco y negro; el frigidarium, la totalidad del muro de mediodía, en el que se abren sendas hornacinas, la mayor con una pequeña bañera a sus pies, y un mosaico, también con decoración geométrica en blanco y negro, compuesta a base de rombos y cruces gamadas inscritos en una cenefa en forma de greca; la cella piscinalis conserva todo su recinto (descubierto durante los trabajos de 1989), con sus dos escalones interiores; el tepidarium o cella tepidaria, 
el pavimento inferior (area) casi intacto, y, parcialmente, el pavimento superior (suspensura) y los pilarcillos de sustentación; las dos estancias cálidas, assa sudatio o sudatorium, restos del pavimento superior, que fue de mosaico (el inferior, que apareció intacto en 1957, se deterioró posteriormente), y la cella caldaria o caldarium, sendas exedras a tramontana y mediodía, cuyas paredes laterales presentan hornacinas, la de levante con restos de un alveus o pequeña bañera en el interior. También se conservan vestigios de los muros que delimitaban a levante y a poniente la dependencia de servicios de los hornos del tepidarium, el caldarium y el sudatorium y de los desagües de las dos últimas estancias.

Al iniciar la intervención en los restos, en 1989, aún no se había trazado un criterio definitivo de cómo hacerla, aunque entonces se barajaba la posibilidad de la reconstrucción ideal, y en ese sentido se hacían estudios. No obstante, se tomaron ya una serie de medidas de consolidación (se recalzaron diversos muros del apodyterium, sudatorium y caldarium, se inmovilizó la base del muro este del tepidarium y se apuntalaron algunos pavimentos antiguos). Algunas de estas actuaciones pudieran ahora parecer opuestas al criterio definitivo (conservar estrictamente lo heredado), pero lo cierto es que eran inexcusables (por ejemplo, la recuperación de la superficie del apodyterium -en buena parte perdida- mediante un pavimento de mortero de cal y arena, lo que se hizo sobre un forjado de viguetas pretensadas y revoltones, con una trampilla que permite el mantenimiento).

Una vez decidido conservar los restos en su apariencia de ruina arquitectónica, lo que implicaba la renuncia a cualquier intento de reconstrucción ideal o de purificación mediante la eliminación de restauraciones anteriores o de los desperfectos que la acción humana y el tiempo habían provocado, se acometieron los trabajos de consolidación de muros y de limpieza y restauración de revestimientos murales y de mosaicos.

\section{La consolidación de muros y revestimientos}

En la exploración de los restos, dificultada por una capa de suciedad que impedía distinguir los materiales, se detectaron sales en los muros (especialmente en los del frigidarium y el tepidarium) y humedad por capilaridad, sobre todo en el suelo de este último. Más tarde, una vez iniciada la limpieza de los muros del caldarium y de la sudatio, se observó la existencia de hongos que, según el informe de la Facultad de Geología, no eran motivo de preocupación al haberse extinguido su ciclo.

La limpieza se realizó en dos fases: la primera fue mecánica, mediante palas, espátulas y paletinas para extraer la suciedad; el polvo levantado se eliminó con pinceles, cepillos y aspiradora; por último, se hizo una limpieza química, actuando sobre los pavimentos y los revoques de opus signinum con agua destilada y cepillos de uñas. La segunda operación fue eliminar las sales de los muros con apósitos de pasta de celulosa con agua destilada. Se llevaron a cabo tres aplicaciones en el frigidarium y en el tepidarium. Se tomó a bofetón una capa de pasta de papel y, una vez seca, se arrancó para arrastrar las sales. De cada aplicación se obtuvo una muestra para hacer, mediante conductímetros eléctricos, un análisis cuantitativo de las sales. Al desprenderse éstas, se pudieron apreciar los

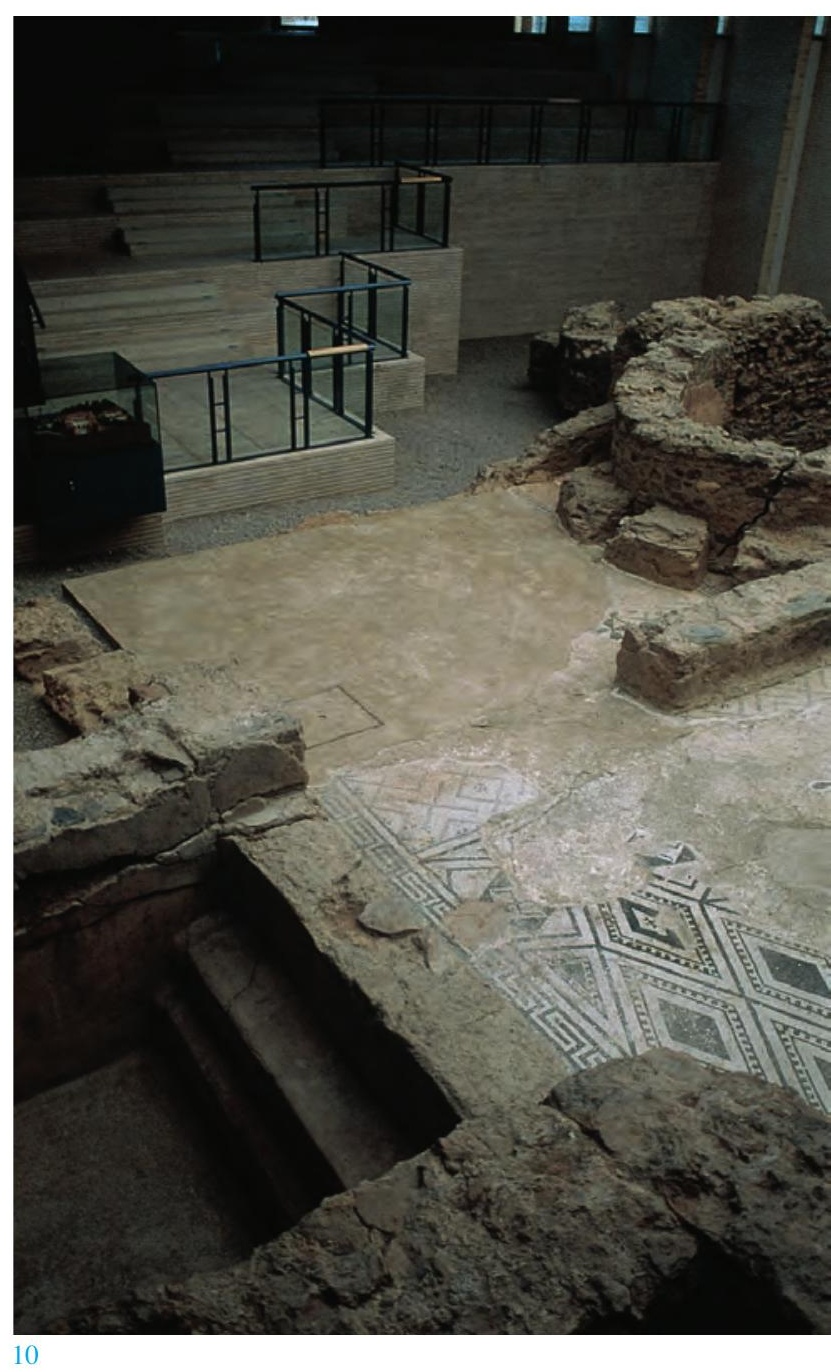

10 

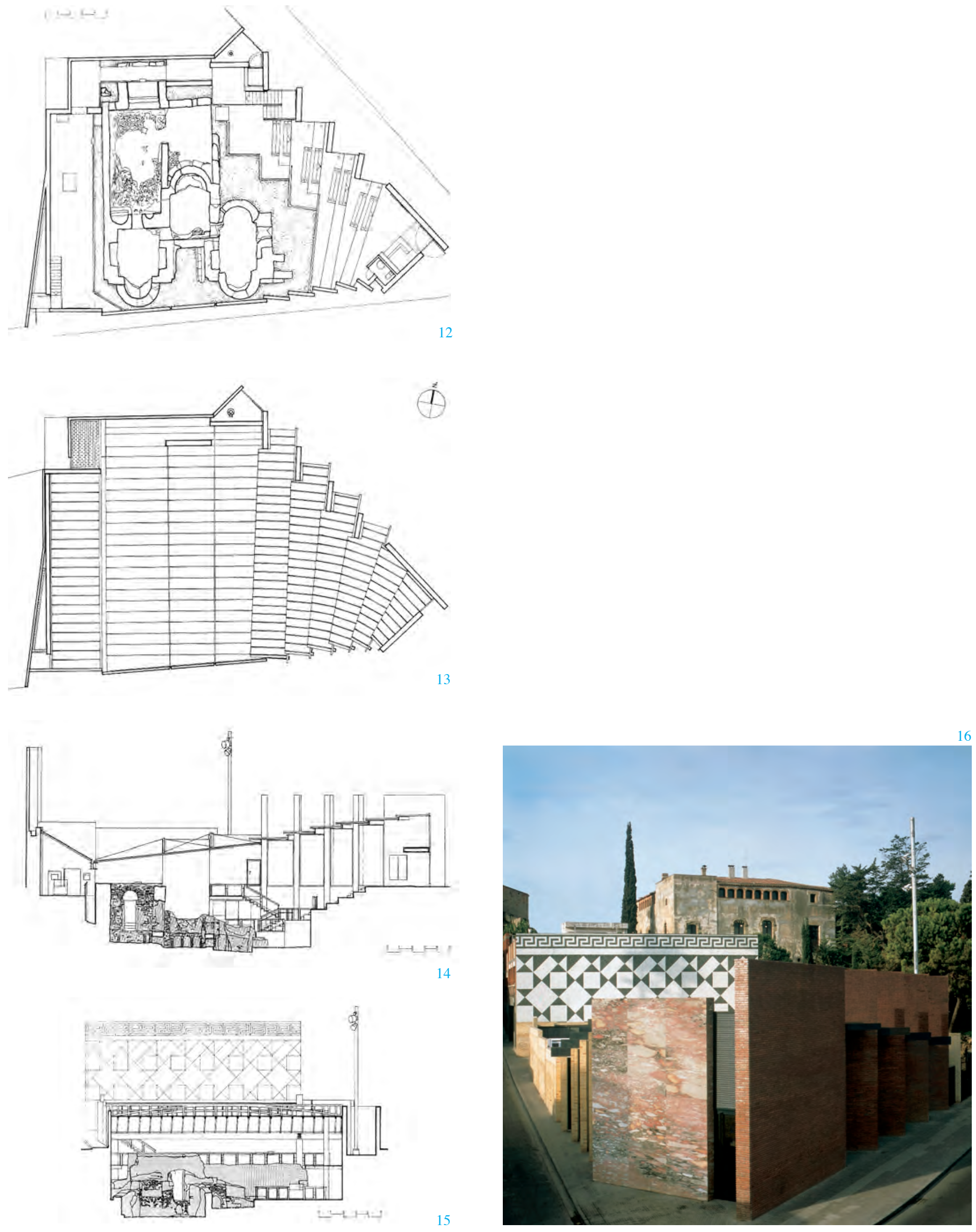

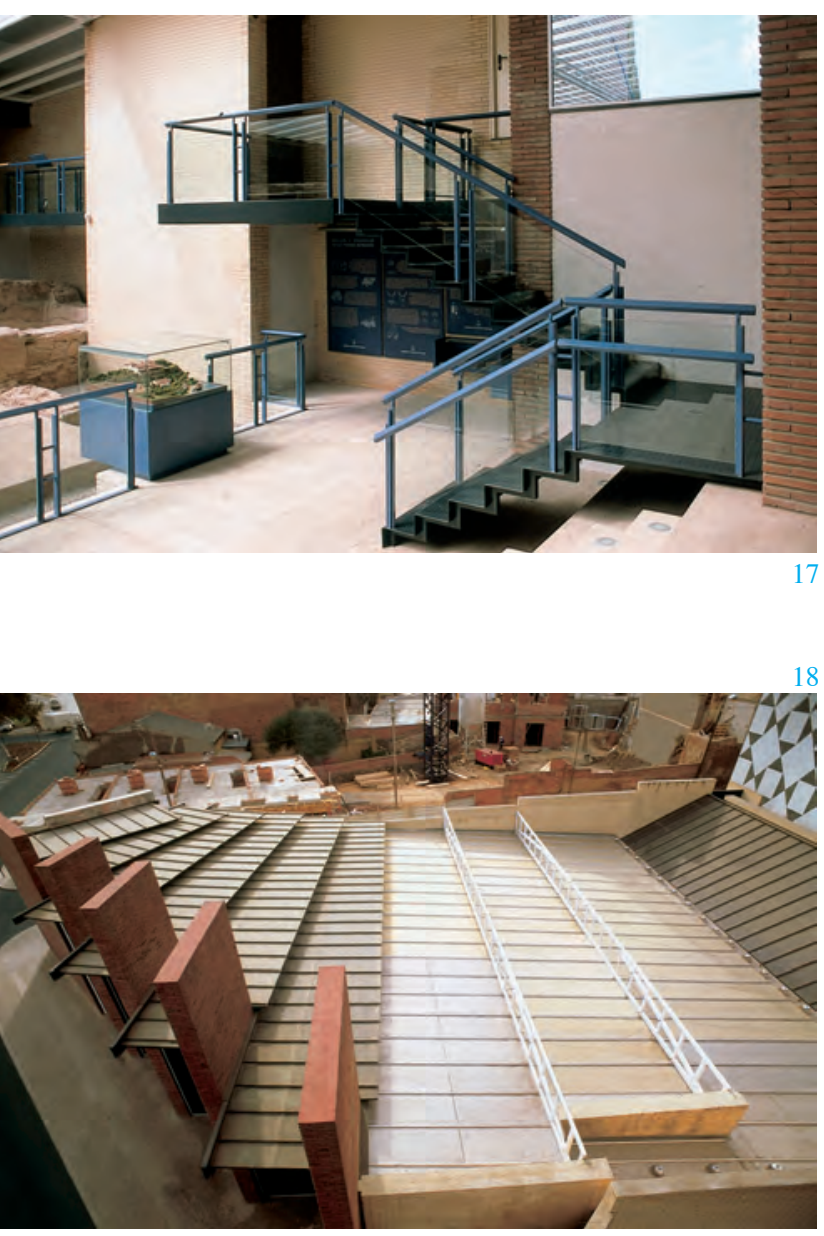

19

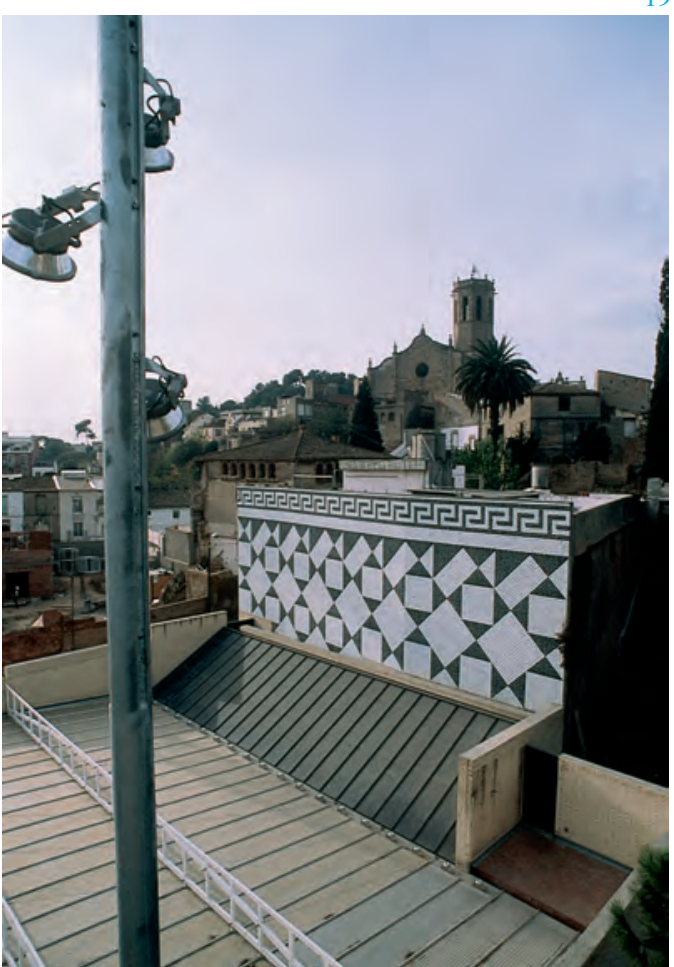

titularidad municipal de los espacios vecinos permitió replantear la línea divisoria de ambas parcelas e incorporar a la nuestra terreno suficiente en el que proyectar una ampliación del edificio en construcción que solucionara algunos problemas no del todo resueltos en el primer proyecto.

El proyecto de ampliación posibilitó redefinir la relación de las ruinas con el edificio (cuyo espacio se enriqueció notablemente), mejorar el recorrido de la visita, y abrir una tercera puerta (apta para el acceso al recinto de personas discapacitadas) y un acceso directo a la cubierta para su mantenimiento, así como excavar y liberar los restos de la piscina, que antes se adentraban en el predio vecino. La redacción del nuevo proyecto también se aprovechó para tratar de subsanar algunos errores técnicos y de planteamiento del primer proyecto (especialmente, la cubierta, con serios problemas de estanquidad, y otros derivados de la falta de ventilación, lo que había obligado a una serie de estudios de climatización) ${ }^{5}$.

El edificio tiene dos fachadas a la calle (a mediodía y levante), una al jardín municipal vecino, al norte, y una medianería con un edificio de viviendas, a poniente. Las tres fachadas están resueltas a base de lienzos ciegos de obra vista, en dos de ellas en disposición oblicua respecto a la alineación de las calles y alternados con cerramientos de vidrio (enmarcados con junquillos metálicos), que permiten la entrada de luz natural y la visión de las termas romanas desde el exterior. Los paramentos de las fachadas laterales son de ladrillo blanco amarillento y los de la fachada principal, de ladrillo rojo manual, de formato catalán. Estos lienzos, que descansan sobre los muros de contención de tierras formando un todo continuo, sobresalen de la línea de coronamiento de las cubiertas a manera de acroterio.

El muro medianero del edificio de viviendas (mucho más alto que el nuestro), está doblado para mejorar su protección frente a agentes atmosféricos. En el paramento externo del nuevo muro, un revestimiento de estuco y pintura con dibujos geométricos y grecas reproduce un detalle del pavimento de mosaico romano conservado y actúa como elemento publicitario e identificador en la trama, visible desde un amplio entorno.

Hay tres accesos directos al interior: el principal, situado en la confluencia de las dos calles, presenta junto a la puerta de vidrio un lienzo de mayor altura que el resto de los paramentos, revestido de placas de mármol de tonalidades rosa, del tipo ArniViola; el segundo (que actúa también como salida de emergencia), se encuentra en el extremo suroeste; y el tercero, situado junto a la medianería del jardín público vecino, está adaptado para el acceso a personas con discapacidades físicas.

\section{La cubierta}

La construcción tiene unos $550 \mathrm{~m}^{2}$ de planta y una altura máxima respecto a la rasante de los viales de unos 5,5 m. Los restos arqueológicos ocupan 175 $\mathrm{m}^{2}$, aproximadamente, tienen casi tres metros de altura de promedio y están a unos cuatro metros por debajo de las rasantes. El interior del edificio es un espacio único continuo que permite la visión constante, itinerante y panorámica de los restos de las termas. La cubierta se desarrolla en tres tramos: el primero es un conjunto de paneles opacos, revestidos de cobre, extendidos a 
modo de abanico escalonado y solapados a distinto nivel, lo que facilita la ventilación del espacio. Se soporta sobre siete vigas que apoyan sobre algunos muros y se anclan lateralmente por el alma a otros.

El segundo tramo, que corresponde a la parte central del edificio, es un plano inclinado, en su mayor parte translúcido, de placas de policarbonato alveolar, lo que permite la iluminación natural diurna y la artificial nocturna de los restos. En las zonas que cubren áreas del recorrido, los paneles son opacos, autoportantes, de plancha pisable de aluminio, sobre algunos de los cuales emergen extractores estáticos de fundición para incrementar la ventilación del espacio interior. La estructura está formada por dos vigas que sobresalen del plano de cubierta, (a modo de vigas en celosía hechas a base de tubos rectangulares), de las que cuelgan las correas, también de tubo de acero, rigidizadas y estabilizadas mediante tensores cruzados de acero inoxidable.

El tercer tramo de la cubierta, también de una sola pendiente, pero de sentido inverso que el del segundo, está resuelto con paneles opacos revestidos con lámina de cobre. Éste y el segundo tramo descansan sobre unas vigas que esconden el canal de recogida de aguas 6 .

\section{Accesos y recorridos}

Por la entrada principal se accede, a pie llano, al vestíbulo y a las gradas por las que se desciende al nivel del yacimiento y desde las que éste puede ser contemplado según diversas perspectivas. Los paramentos verticales de las gradas están revestidos con losas de mármol travertino clásico, con acabado de sierra, decoradas con acanaladuras horizontales. El pavimento es, como en el resto de espacios, de mármol travertino sin pulir y los escalones de las gradas se han construido con hormigón prefabricado visto. Los espacios libres entre las ruinas y la obra nueva están pavimentados con grava de tipo ojo de perdiz, a modo de alfombra. En el extremo opuesto a la entrada, junto a la medianería, se sitúa una sala rectangular, abierta hacia los restos, lo que permite su visión desde una altura intermedia. En el ángulo suroeste de esta sala, un pequeño balcón triangular vuela para poder contemplar mejor aquel sector de las termas.

Entre el nivel inferior de las gradas, casi a la misma cota que la de los restos, y esa sala intermedia discurre el recorrido lateral: se inicia con una escalera metálica que alcanza un balcón sobre los restos, del que parte una pasarela horizontal que conecta con aquella sala oeste. Desde ese nivel del balcón, una plataforma móvil permite el acceso y la salida de personas discapacitadas, que pueden continuar el recorrido a través de la pasarela. La estructura de este elemento y el balcón del recorrido de visita se compone de pasamanos de 240x40 mm y rigidizadores de 70x20 mm. El pavimento es de chapa de acero autoportante, con relieve, pintada al esmalte, como en el caso de la escalera. Las barandillas y protecciones han sido ejecutadas con pasamanos y montantes de acero y lienzos de vidrio laminar y lámina de butiral. Los pasamanos son tubos de sección cuadrada, con una de sus diagonales en vertical. En los lugares en que la contemplación de las ruinas resulta más explicativa o sugerente, para inducir al espectador a detenerse hay posabrazos de madera de haya barnizada de sección semicilíndrica.
17. Escaleras de comunicación entre las diversas plataformas creadas para la contemplación de las termas desde perspectivas diferentes, siguiendo un itinerario circundante (foto: Montserrat Baldomà. SPAL. 10.09.1998)

18. Vista exterior de la cubierta del edificio de protección de las termas (foto: Montserrat Baldomà. SPAL. 25.11.1998)

19. Torre de luz que ilumina de noche el interior del edificio a través de la lucerna (foto: 25.11.1998)

20. Visión de la plataforma elevada del sector de poniente del edificio de protección (foto: Montserrat Baldomà. SPAL. 10.09.1998)

21. Plataforma situada a nivel del acceso al apodyterium (foto: Montserrat Baldomà SPAL. 10.09.1998)

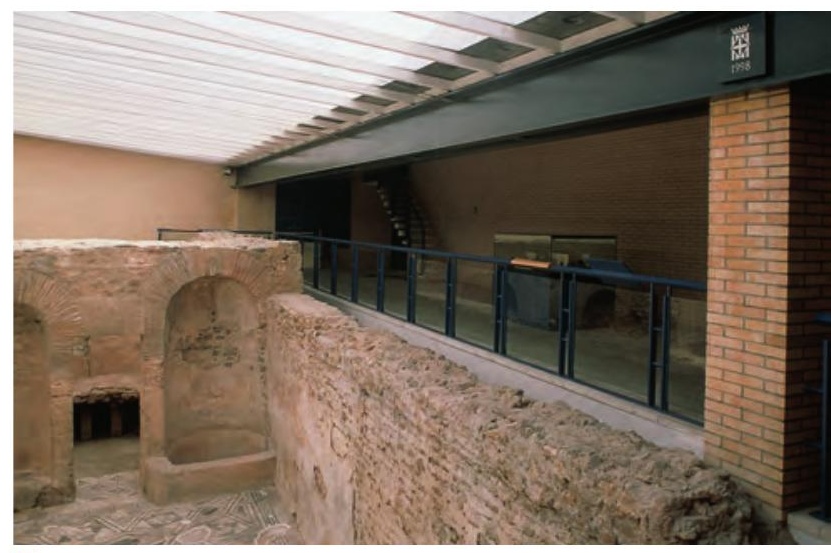
20

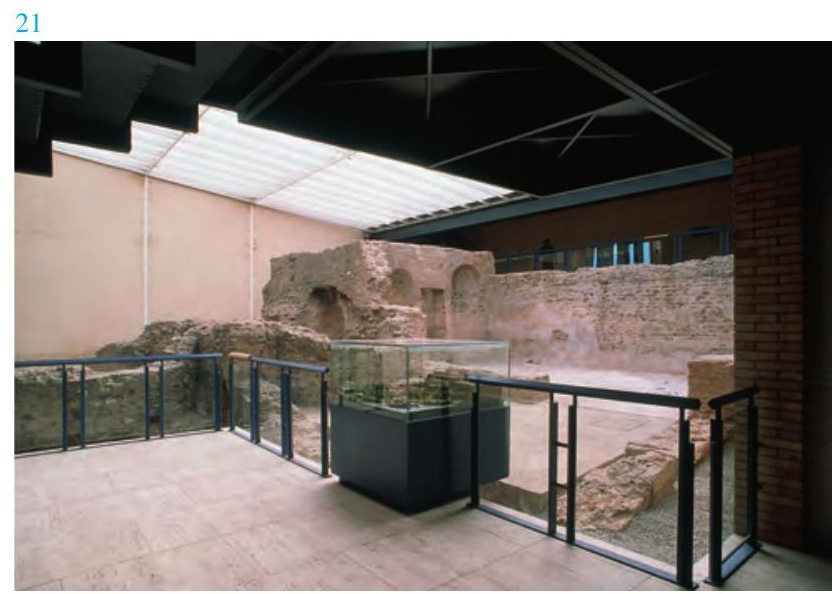


"usted está aquí" señalado por un punto rojo sobre el vidrio de protección. A lo largo del recorrido posterior, dos atriles muestran diferentes vistas de las termas (orientadas como éstas, para no confundir al espectador) indicando las diversas salas. En la segunda maqueta se recrea el edificio termal en uso, seccionado, para que el visitante pueda comprender el funcionamiento de las diversas salas. Al final del itinerario, un panel informa de la actividad del centro productor de ánforas que existió en el lugar antes de la construcción de las termas, acompañado de una vitrina con fragmentos de ánforas y la explicación de las marcas de los alfareros, y de un ánfora recuperada y restaurada en los años sesenta, colgada en la pared sobre un panel.

La iluminación artificial de las ruinas procede de un elemento exterior que las baña a través de la lucerna, como si de luz natural se tratara. En el interior, unos focos empotrados en el suelo subrayan la volumetría y el trazado de las gradas y sus escalones. La sala oeste no cuenta con alumbrado específico, al ser suficiente el procedente de las ruinas iluminadas.

\section{Notas:}

1. Documento escrito por el servita fray Pere Puig conservado en el Archivo Parroquial de Sant Boi

2. Josep de Calassanç Serra Ràfols era conservador del Museo Arqueológico, dependiente de la Diputación de Barcelona, y, en aquel momento, Comisario Provincial de Excavaciones

3. Denominación que tuvo hasta 1987 el actual Servicio de Patrimonio Arquitectónico Local de la Diputación de Barcelona

4. Para más información sobre las termas de Sant Boi y la intervención del SPAL, consultad: «Edificio de protección de las termas romanas. Sant Boi de Llobregat», en A. González, La restauración objetiva. Método SCCM de restauración monumental. Memoria SPAL 1993-1998, vol. 2, pág. 261, Barcelona, Diputación de Barcelona, 1999; Albert López Mullor, Imma Estany: «L'excavació a l'àrea de les termes romanes de Sant Boi de Llobregat» (campanyes 19891991), en Quaderns Científics i Tècnics, 5, Diputación de Barcelona, 1993; Raquel Lacuesta: La intervenció de 1953-1975 a les termes romanes de Sant Boi de Llobregat (Barcelona). SPAL, 1999 (en prensa)

5. No todos los errores pudieron ser resueltos totalmente. Está prevista para el año 2000 una revisión general de la obra, cuando, doce meses después de haber entrado en uso, se tengan más datos sobre su comportamiento real. Con objeto de tener constancia de la evolución de la temperatura y la humedad del interior del recinto a lo largo del año y en función de los diferentes estadios de uso del edificio, en el verano de 1998 se colocaron en cinco puntos estratégicos sendos termohigrógrafos con control remoto por ordenador. El estudio de los resultados permitirá, en caso de que se demuestre que la realidad ambiental actual puede perjudicar la buena conservación del yacimiento o la comodidad de los visitantes, que se hagan las correcciones necesarias

6. Acabadas las obras, pronto se detectaron problemas derivados del deficiente diseño de la cubierta en el primer proyecto, problemas que no pudieron resolverse del todo en el curso de la obra. El día de la inauguración, el 11 de septiembre de 1998, diada nacional de Cataluña (una fecha de especial significación en Sant Boi por estar enterrado en su iglesia parroquial Rafael de Casanovas, que aquel mismo día, 284 años antes, capitulara en Barcelona frente a las tropas del rey), llovió con la furia propia de la estación. Mientras el presidente de la Diputación leía su discurso, un curso de agua intermitente e impertinente, que se había abierto paso entre los materiales aún no suficientemente hermanados de la cubierta, rozaba las cuartillas que sostenía entre sus manos
22. Maqueta de recreación ideal de la villa romana de Sant Boi (foto: Montserrat Baldomà. SPAL. 10.09.1998)

23. Paneles informativos y vitrinas con una selección de los materiales arqueológicos hallados en diversas campañas de excavación (foto: Montserrat Baldomà. SPAL. 10.09.1998)

24. El recorrido perimetral de las termas, creado mediante escaleras y pasarelas, ofrece al espectador diferentes puntos de mira, insinuados por los posabrazos de madera de las barandillas (foto: Montserrat Baldomà. SPAL. 10.09.1998)

25. Visita nocturna del interior del edificio, siguiendo el recorrido previsto en el proyecto museístico (foto: Montserrat Baldomà. SPAL. 10.09.1998)

\section{FICHA TÉCNICA}

LOCALIZACIÓN: Sant Boi de Llobregat (comarca, Baix Llobregat; provincia de Barcelona)

ACTUACIÓN: Promovida por el Ayuntamiento y realizada por el SPAL de la Diputación de Barcelona (1989-1998) INVESTIGACIÓN HISTÓRICA:

Fuentes documentales: Raquel Lacuesta

Arqueología: A. López, I. Estany, X. Fierro, À. Caixal

Numismática: María Clua

Paleofauna: Lluís Rius

Prospección geofísica: Lluís Marí, Josep M. Niñerola

Estudio materiales constructivos y patologías: M. Vendrell EDIFICIO DE NUEVA PLANTA:

Primer proyecto: Andreu Arriola y Carme Fiol, arquitectos Mejora y ampliación: Antoni González, arquitecto

Colaboradores: P. Carbó, J. Rovira, J. M. Sala, S. Blasco, arquitectos; A. Elizondo, J. Bassas y A. Martí, aparejadores;

J. Corderroure, maestro de obras

CONSOLIDACIÓN DE LAS TERMAS:

Coordinación: Francisco J. Asarta, arquitecto

Revestimientos y mosaicos: A. Cusó, N. Zapata, L. López, M. Alcobé, M. Buchanan

\section{MUSEIZACIÓN:}

Programa: Gregorio Gutiérrez y Raquel Lacuesta

Diseño: Olga de la Cruz. Grafismo: Quim Boix

Maquetas: Grau-Alcázar

INVERSIÓN TOTAL: 238.000.000 PTA.

Tratamiento de los restos: $53 \mathrm{~m}$. (excavación arqueológica, 17,5; restauración, 24,5; estudios y trabajos: 11)

Edificio de protección: 185 m. (obra: 165; estudios y trabajos: 20) 